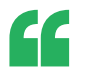

deletion of

AKAP220

... resulted

in impaired

actin-barrier

formation

and over-

accumulation

of AOP2

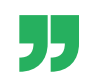

\title{
Vasopressin-independent AQP2 trafficking
}

New data suggest a role of AKAP220 in the vasopressin-independent regulation of AQP2 trafficking and water homeostasis. Howard Hughes Investigator John D. Scott and colleagues suggest that their findings could have clinical relevance for patients with nephrogenic diabetes insipidus, who are unable to concentrate their urine in response to vasopressin.

"We discovered AKAP220 as a PKA-anchoring protein in 1996," says Scott. "Our interest in this protein grew with the discovery of more signalling enzyme-binding partners, including PP1 and GSK3, and of a link between the AKAP220 complex and the cytoskeletal scaffold protein IQGAP. Further work showed that elements of the AKAP220 complex confer cAMP-mediated control of cytoskeletal remodelling. The discovery that AKAP220 associates with the AQP2 water pore set the stage for our current study led by Jennifer Whiting."

To investigate the role of AKAP220 in water homeostasis, the researchers used CRISPR/Cas9 gene editing of organoid cultures derived from murine inner medullary collecting duct cells, AKAP220-knockout mice and super-resolution imaging approaches. They found that deletion of AKAP220 disrupted the apical actin network of the organoids, and
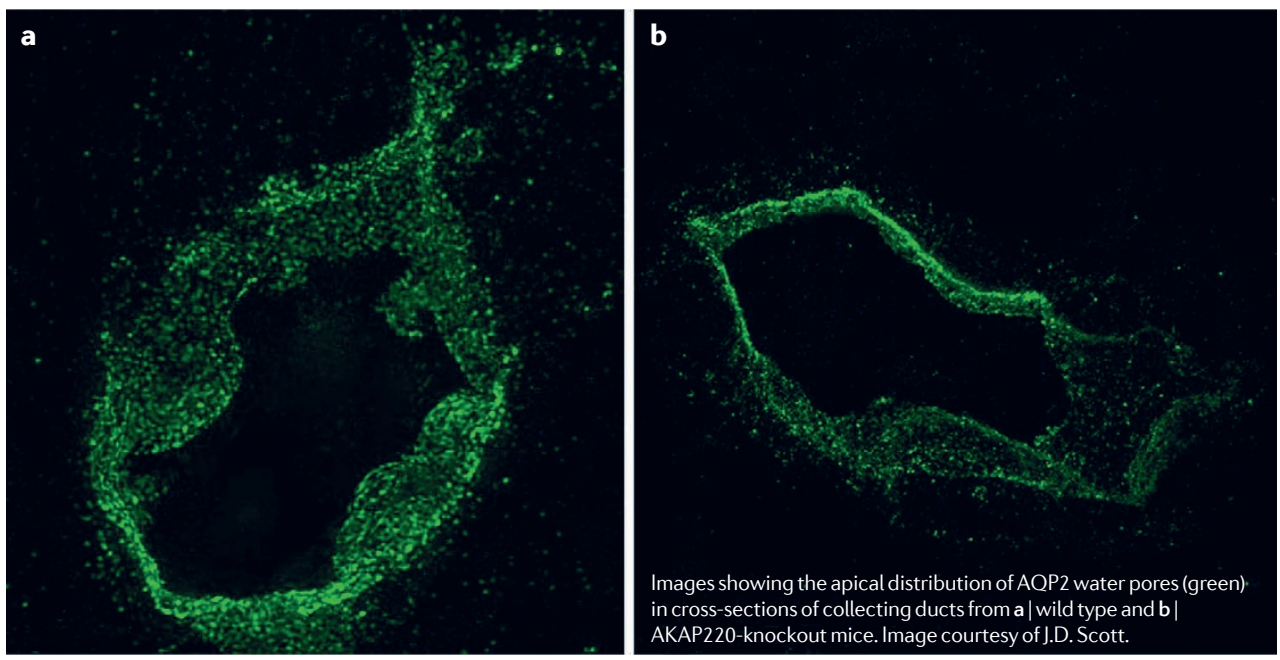

resulted in impaired actin-barrier formation and over-accumulation of AQP2 and RhoA — a modulator of the actin cytoskeleton - at the apical surface of collecting duct cells in the knockout mice. Moreover, when overhydrated, these mice excreted urine with an abnormally high osmolality, indicating a defect in urine-diluting capacity (that is, inappropriate water reabsorption) in response to overhydration.

"These findings point toward the AKAP220/IQGAP axis as a nexus for the regulation of actin dynamics in kidney cells," says Scott. "The formation of large AKAP220 macromolecular assemblies provide a means to integrate and focus distinct cellular signals at the apical membranes of the collecting duct cells."

The researchers have now used CRISPR/Cas9 technology to generate mutant forms of AKAP220 that lack the ability to bind individual enzyme-binding partners. They plan to focus their future studies on the role of AKAP220-anchored PP1 in the modulation of AQP2 trafficking and membrane insertion.

Ellen F. Carney

ORIGINAL ARTICLE Whiting, J. L. et al. AKAP220 manages apical actin networks that coordinate aquaporin-2 location and renal water reabsorption. Proc. Natl Acad. Sci. http://dx.doi. org/10.1073/pnas.1607745113 (2016) 